\title{
Experimental and computational investigation of kinematic mixing in a periodically driven cavity
}

\author{
S. Santhanagopalan, A. P. Deshpande \& S. Pushpavanam \\ Department of Chemical Engineering, \\ Indian Institute of Technology Madras, India
}

\begin{abstract}
Mixing of fluids is important in many industrial processes. In this paper we discuss the process of kinematic mixing in Newtonian fluid and quantify the intensity of mixing. A cavity with a periodically driven lid was chosen for the experimental and numerical analysis. It is shown that the system behavior depends on two dimensionless parameters, the geometric parameter $\left(\mathrm{A}_{\mathrm{f}}\right)$ and the Stokes number $(\mathrm{St})$. The mixing is quantified by the Planar Laser Induced Fluorescence (PLIF) technique using Rhodamine-B as the dye. Here the emitted fluorescent light intensity was related to the concentration of the dye present in the system. The extent of mixing was determined by calculating the deviation of intensity fluctuations. In addition, numerical analysis using the Particle Separation approach based on the calculation of stretch rates were done and applied to study the effect of two dimensionless parameters on mixing.
\end{abstract}

Keywords: mixing, periodically-driven cavity, Planar Laser Induced Fluorescence, stretching.

\section{Introduction}

Mixing is an important feature in many chemical processes. In a chemical reactor a reaction between two or more reactants can occur only when they mix with each other. Chien et al. [1] studied qualitative laminar mixing in a twodimensional cavity under different configurations based on the motion of the walls. They used material line and blob deformation for their analysis. They found that efficiency of mixing depends strongly on the frequency of oscillation of the walls and reported an optimum value of frequency, which produces best 
mixing in a given time. They also found that the alternate periodic motion of the two walls yields chaotic mixing and demonstrated the existence of Horseshoe functions as an indication of chaotic behavior.

Leang and Ottino [2] carried out flow visualisation experiments on mixing at low Reynolds numbers. The periodic flow was induced in the cavity by means of continuous motion of the wall. They identified periodic points and reported the symmetric occurrence of these points at regular intervals.

Anderson et al. [3] found a method to locate periodic structures in three dimensional time-periodic flows and applied this to mixing in cavity flows. They numerically obtained velocity and stretching fields to identify the periodic points. They tested this method for different mixing protocols.

Chaotic mixing in a bounded three-dimensional flow at moderate Reynolds numbers was investigated by Fountain et al. [4]. The flow structures were captured using a fluorescence dye. They also compared the experimental investigations with numerical simulations and predicted the number of periodic islands.

Roberts and Mackley [5] characterised the kinematic mixing rates quantitatively by computing stretching rates using numerical techniques. The mixing rates were evaluated for two cases i) constant volumetric flow and ii) oscillatory flow in a baffled channel. The mixing rates obtained were based on using a particle separation approach as well as a line element approach. They determined the stretch rate dependency on Reynolds number as well as how the stretch rate varied over the entire flow field.

Classically mixing has been characterised using stretch rates. The repeated stretching and folding is an indication of the amount of mixing. The Lyapunov exponent of a dynamical system is analogous to the stretch rate in a fluid mechanical system. The key to effective mixing lies in producing repetitive stretching and folding.

The mixing that we discuss in this work arises primarily due to convective flow. We neglect, mixing due to diffusion or dispersion. Here we investigate the mixing of Newtonian fluid in periodically driven cavity arrangement. We experimentally quantified mixing by calculating the deviation in the intensity fluctuations. The mixing is also quantified from numerical simulations based on calculation of stretch rates.

\section{Quantification of mixing from PLIF measurements}

\subsection{Experimental details}

Fig. 1 shows a front view of the cavity (A) and the working model. The top plate (B) was designed to move to and fro along the $\mathrm{x}$-direction. This was rendered possible with a guide plate $(\mathrm{C})$ arrangement. The periodic motion of the top plate was achieved with a drive wheel (D) and connecting rod (E) assembly. Ball bearings (F1, F2) were used for attaching each end of the connecting rod as well as to support the drive wheel. A permanent magnet DC motor $(\mathrm{G})$ was used to turn the drive wheel. The speed of the motor was controlled by a thyristor drive $(\mathrm{H})$. The plate frequency was varied using the thyristor drive. The amplitude 
(maximum displacement) of the top plate motion was set by the radius at which the connecting rod was attached to the drive wheel. For this several holes at different radial positions were provided in the drive wheel (D).

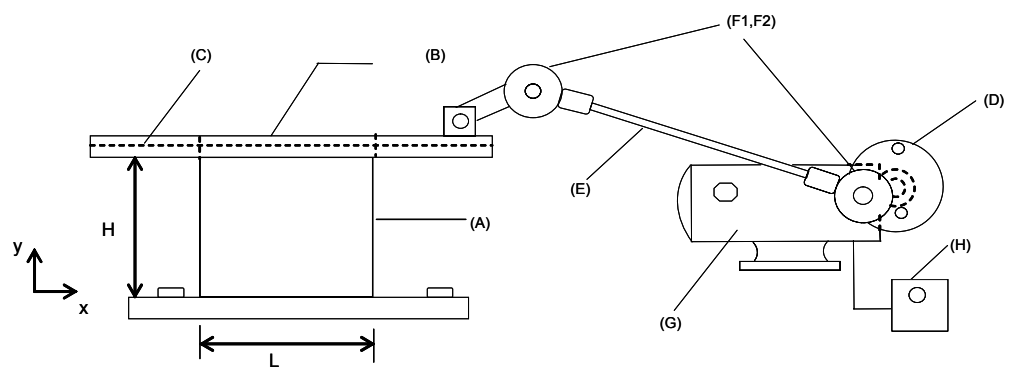

Figure 1: $\quad$ Schematic diagram of the experimental setup.

The dimensionless Reynolds number is defined as $\mathrm{Re}=\left(\mathrm{U}_{\max } \rho \mathrm{H}\right) / \mu$ and the Stokes number is defined as $\mathrm{St}=\omega \rho \mathrm{H}^{2} / \mu$. Here the maximum velocity of the plate $\left(U_{\max }=A \omega\right)$ is the velocity scale, $\rho$ is the fluid density and $\mu$ is the viscosity of the fluid. The Strouhal number (Str) is defined as $\mathrm{H} \omega / \mathrm{U}_{\max }$. Since in our system $U_{\max }=A \omega$, Strouhal number get reduced to the amplitude factor $A_{f}(=$ $\mathrm{H} / \mathrm{A}$ ). The penetration depth i.e. the distance up to which the momentum penetrates in the cavity in relation to the depth of the cavity is an important parameter. So depth of the cavity $(\mathrm{H})$ is chosen as the length scale for our problem. The choice of Stokes number and the Amplitude factor has the advantage that we can independently study the effects of the amplitude of the plate motion by varying $\mathrm{A}_{\mathrm{f}}$ keeping $\mathrm{St}$ constant and the frequency of the plate motion by varying $\mathrm{St}$ keeping $\mathrm{A}_{\mathrm{f}}$ constant.

\subsection{Experimental technique}

The mixing process was studied by Planar Laser Induced Fluorescence (PLIF), using Rhodamine-B as the fluorescent tracer. The tracer solution of $0.5 \mathrm{ml}$ was injected into the cavity after the periodic state is achieved. The central plane of the mixing zone $(0.096 \mathrm{~m} \times 0.08 \mathrm{~m})$ was illuminated using $1 \mathrm{~mm}$ thick $\mathrm{Nd}$ : YAG laser sheet, of $120 \mathrm{~mJ}$ pulse energy and $532 \mathrm{~nm}$ wavelength. The light emitted from the fluorescent molecules was recorded using FlowMaster-3S charge coupled device (CCD) camera (1280 x 1024 pixels, $8 \mathrm{~Hz})$ from La Vision. The acquisition rate was $4 \mathrm{~Hz}$. Two dimensional single frame PLIF images were taken at different time intervals. In order to eliminate the overlapping effect of illumination and emission, a high-pass optical filter (with a cut off wavelength of $550 \mathrm{~nm}$ ) was placed in front of the camera to obtain the fluorescence signal rejecting the laser light.

\subsection{Calibration measurements}

The calibrations were done by performing measurements on homogeneous solutions of known concentrations. The amount of emitted light captured on the 
CCD camera is a function of the concentration of Rhodamine-B. For each concentration 50 images were taken and the average value of intensity was found. Figure 2 shows the linear relationship between the averaged fluorescent intensity and the Rhodamine-B concentration. For the extreme values of concentration $\mathrm{C}_{\min }$ and $\mathrm{C}_{\max }$ the intensity represented as $\bar{I}_{\min }(i, j)$ and $\bar{I}_{\max }(i, j)$ was calculated. The normalized fluorescence intensity fields were calculated based on,

$$
G_{i}(i, j)=\frac{I_{i}(i, j)-\mathrm{I}_{\min }(i, j)}{I_{\max }(i, j)-\mathrm{I}_{\min }(i, j)}
$$

The dye was injected into the cavity after the initial transients had decayed. The PLIF images were taken at different time intervals for total mixing time of $720 \mathrm{~s}$.

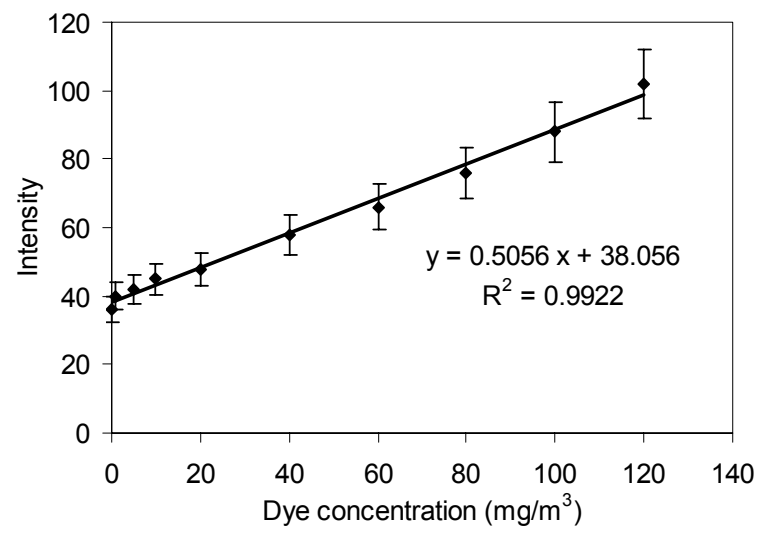

Figure 2: Fluorescence intensity as a function of dye concentration.

The extent of mixing is quantified by calculating the deviation of intensity given by,

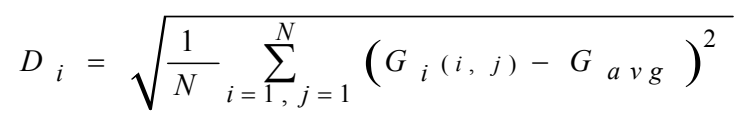

where $\mathrm{G}_{\mathrm{i}}$ and $\mathrm{G}_{\mathrm{avg}}$ denote the normalised intensity at pixel $(\mathrm{i}, \mathrm{j})$ and the maximum intensity, respectively.

\subsection{Effect of dimensionless parameters on mixing}

During a typical mixing run, the dye was injected after the system had an attained periodic state. Instantaneous images were taken at different time intervals and the deviation in the fluorescence intensity as a function of time for different Stokes number is plotted in Fig. 3. The larger values of the standard 
deviation in the beginning of the experiments show low degree of mixing. As the time progresses the concentration of the dye decays and as a result we see decrease in the standard deviation $\left(\mathrm{D}_{\mathrm{i}}\right)$ showing high degree of mixing. Similar trend was observed for different Amplitude factor and plotted in Fig. 4.

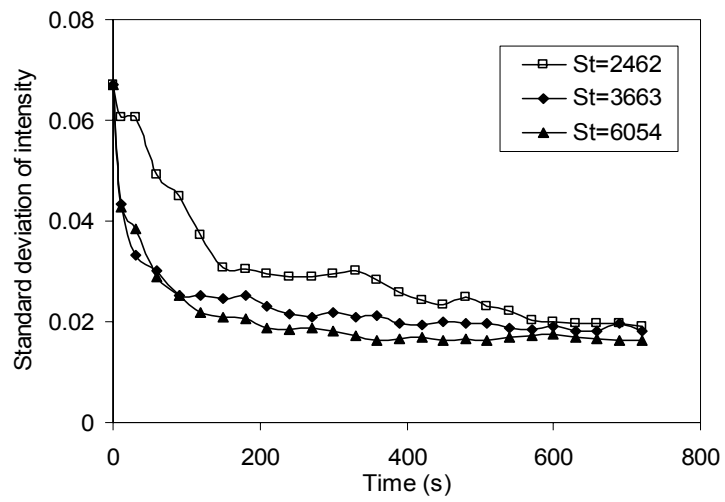

Figure 3: Standard deviation of dye intensity as a function of time for different St.

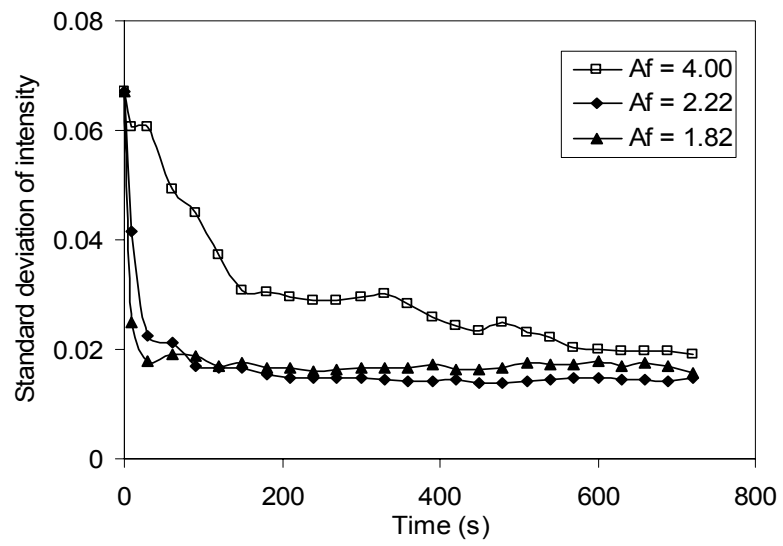

Figure 4: Standard deviation of dye intensity as a function of time for different $\mathrm{A}_{\mathrm{f}}(\mu=0.00473$ Pa.s $)$.

The deviation of the intensities $\left(\mathrm{D}_{\mathrm{i}}\right)$ after $720 \mathrm{~s}$ for different experimental conditions is plotted in Fig 5 and 6. The dependency of Stokes number on $\mathrm{D}_{\mathrm{i}}$ i.e. measure of mixing, is shown in Fig. 5. The concentration of the dye decays much more rapidly at high $\mathrm{St}$ than at low $\mathrm{S}_{\mathrm{t}}$. As a result we see the increase in degree of mixing with increase in St. The dependency of $A_{f}$ on $D_{i}$ is shown in Fig. 6. Here we see that the degree of mixing increases with decrease in $A_{f}$. 


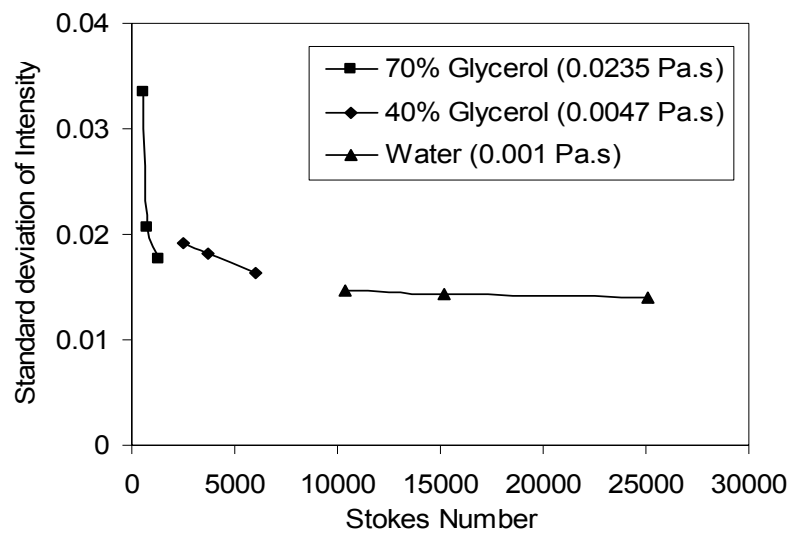

Figure 5: Standard deviation of dye intensity as a function of St.

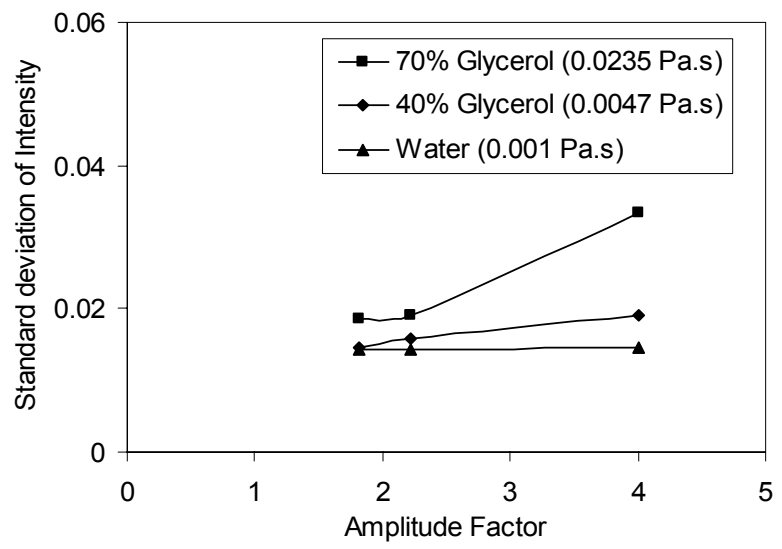

Figure 6: Standard deviation of dye intensity as a function of Af.

\section{Quantification of mixing from numerical computations}

\subsection{Governing equations}

The flow is governed by Continuity equation (1) and Navier-Strokes equation (2). The system of equations is made dimensionless so that we can study the effect of various parameters.

$$
\begin{gathered}
\nabla \cdot \mathbf{u}=0 \\
\frac{\partial \mathbf{u}}{\partial t}+\frac{1}{S t r}(\mathbf{u} \cdot \nabla \mathbf{u})=\frac{-1}{S t r}(\nabla \mathbf{p})+\frac{1}{S t} \nabla^{2} \mathbf{u}
\end{gathered}
$$


where $\mathbf{u}=(u, v)$ is the non-dimensional velocity and $\mathbf{p}$ the non-dimensional pressure. In the above system of equations the reference scales of the length, time, velocity and pressure were $\mathrm{H}, \omega^{-1}, \mathrm{U}_{\max }, \rho \mathrm{U}^{2}$ max .

\subsection{Computational technique}

The fluid motion was tracked by carrying out CFD simulations using Fluent 6.2. The 2-D geometry was made using Gambit 2.1. The grid independency was ensured by meshing the geometry with different spacing between the cells. The quad map type grid with 50000 cells was chosen for detailed studies. A periodic boundary condition on the top plate was imposed using an externally defined macro and the other faces of the cavity were considered as solid stationary walls. The convective terms were discretized using a second order upwind scheme. The coupled implicit time formulation was used with second order accuracy. The particle tracking was done using discrete phase model. Neutrally buoyant tracer particles were injected in the flow domain at different spatial locations, after the system had attained periodic state. The trajectory of a discrete phase particle was obtained as a function of time.

In the present study the particle properties were chosen to be the same as the fluid properties. Therefore the additional forces acting on the particle is zero and the flow is only due to the motion of the plate. The particle was tracked with the fluid flow and time step used was 0.1s. The particles were injected in the flow only after the initial transients have decayed and the flow attains periodic steady state.

\subsection{Particle separation approach}

A Lagrangian approach is used to characterise the mixing process by calculating the stretch rates of the fluid elements. The stretch rate of a small line between two particles can be estimated form the separation rate of adjacent particles, provided the distance between the two particles is small. We now describe a numerical method which allows us to obtain the stretch rates over the entire flow field using an efficient method. The usual algorithm involves tracking the separation of two particles which are close by initially. Once they are separated we inject a second particle close to the new position of the first particle and continue the procedure.

The particles were initially distributed uniformly in the flow domain as shown in fig. From the location of particles of the particles obtained from the particle tracking at different time instants, the distance between two specific particles is calculated at every time instant. The rate of change of distance between the two particles is assumed to be proportional to the instantaneous distance between them. Under these conditions the separation between two adjacent particles initially at a distance of $\mathrm{l}_{\mathrm{o}}$ is given by,

$$
s=\frac{1}{t} \ln \left(\frac{l_{t}}{l_{o}}\right)
$$


where $l_{t}$ is the distance separating them at a time instant $t$ later, shown in Fig.7. This approach however yields the stretch rate at a particular point or region of the flow field. In order to average the stretch rate over the whole flow field the value of $s_{p}$ for all adjacent pairs of particles is determined and their average is calculated.

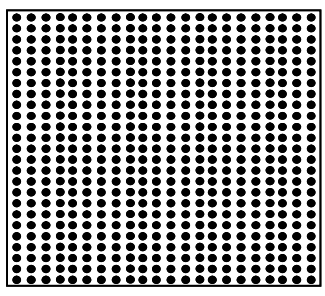

(a)

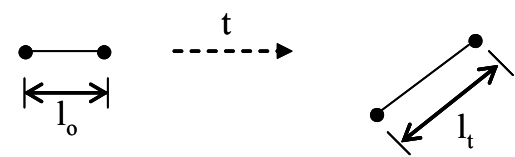

(b)

Figure 7: (a) Schematic showing the initial position of the particle array (b) Stretching of a small line between adjacent particles.

To determine the average stretching rate, 2500 particles were injected in the cavity once the initial transients had decayed and the flow attained a periodic state. These particles were initially in an array of uniform spacing i.e., $50 \times 50$. The motion of individual particles was tracked over a period of time. Using the instantaneous positions of the particles the distance between two particular particles was found and from this the stretch rate was calculated.

The key numerical parameter for the particle separation approach is the number of particles injected as this control the initial separation of the particles, $1_{0}$. Taking the value of $S_{\text {avg }}$ after three periods of cycle for $\mathrm{St}=2024$, the effect of the number of particles was studied. The average stretch rate is independent of the number of particles, provided more than 2000 particles were used.

\subsection{Influence of dimensionless parameters on average stretch rate}

Fig. 8 shows the dependence of $\mathrm{S}_{\text {avg }}$ on time period for different $\mathrm{St}$. The value at the end of every integral multiple of the time period is shown in the Fig. 8. The initial value is close to zero, reaches a maximum value and after this the average stretch rate begins to decrease. For further calculations of stretch rate are based on the values determined at the time period when the stretch rate is a maximum.

The variation of average stretch rate with Stokes and Amplitude factor are shown in Fig. 9 and 10. We observed that the average stretch rate increases with increase in Stokes number. Here the other parameters, amplitude and viscosity were fixed. On the other hand the average stretch rate decreases with Amplitude factor, in which case the angular frequency and viscosity were fixed. In the range of parameters under study, the amplitude of the plate motion shows strong effect on average stretch rates than the frequency, which can be observed from Fig. 9 and 10 . 


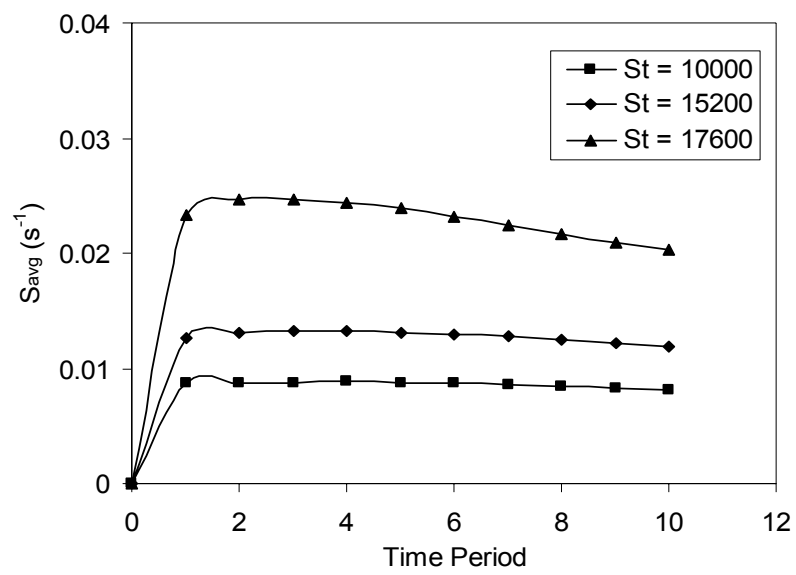

Figure 8: $\quad$ Average stretch rate as a function of time at different St.

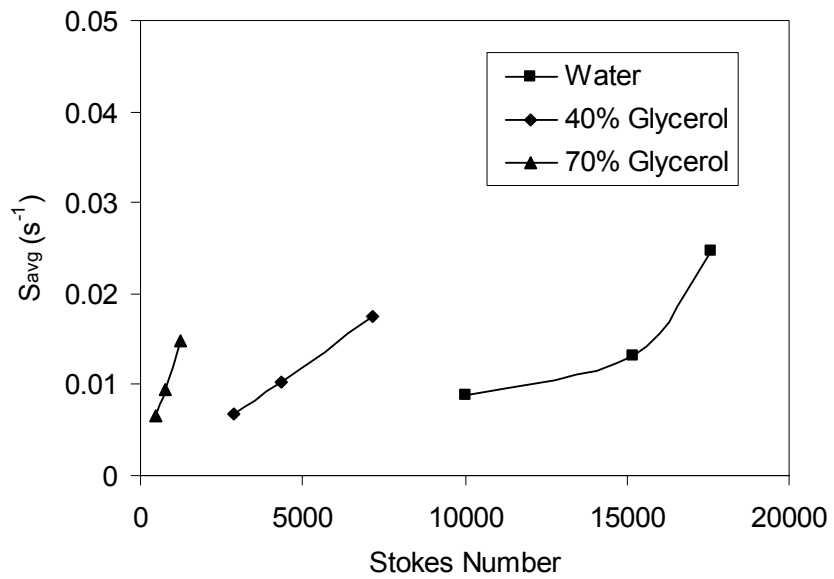

Figure 9: Variation of Average stretch rate on St.

Mixing is efficient when the flow is periodic. In order to compare the order of stretch rates obtained incase of periodic flow, it is compared with flow in a steady lid-driven cavity. To achieve this we define the plate velocity as, $v_{p}=v_{o}+A \omega \sin (\omega t)$. Here $\mathrm{v}_{\mathrm{p}}$ is the plate velocity and $\mathrm{v}_{\mathrm{o}}$ is the maximum plate velocity, which is independent of the frequency as well as the amplitude of plate motion. The equation reduces to $v_{p}=v_{o}$, when the amplitude of motion, $\mathrm{A}=0$ and therefore the flow becomes steady lid driven case.

The simulations were done by varying the amplitudes of motion from $0-0.06$ $\mathrm{m}$ by keeping the other parameters constant. As we see from Fig. 11, the average 
stretch rates are higher, when the flow is periodic which goes through a maximum. In case of steady flow the stretch rate is smaller when compared to PDC and are constant, as the particles follows closed circular path and continue to move in same path.

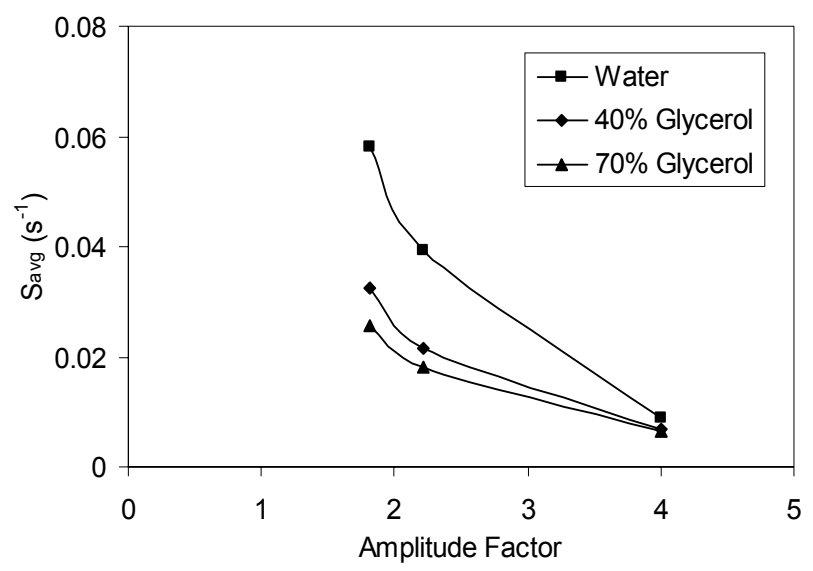

Figure 10: Variation of Average stretch rate on Af.

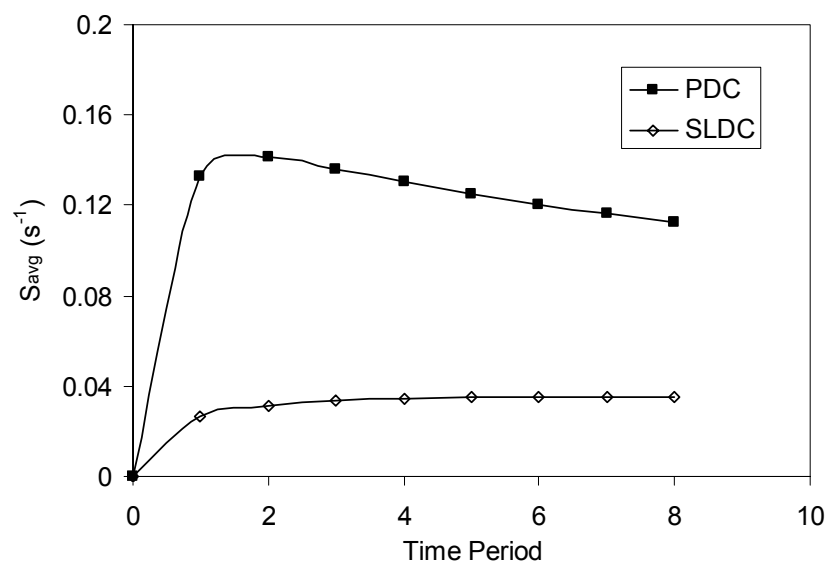

Figure 11: Comparison of average stretch rates for PDC and SLDC.

\section{Conclusions}

The mixing of Newtonian fluid in a periodically driven cavity has been studied experimentally using PLIF technique. Based on the deviation in intensities at every pixel, the extent of mixing at different experimental conditions was calculated and reported. The dependency of the deviation from mixing on the 
dimensionless parameters was studied. The mixing rates were numerically calculated based on stretch rates using particle separation approach. The dependency of the stretch rates on the dimensionless parameters was also studied. It is found that the mixing increases with the Stokes number and decrease with Amplitude factor. The mixing rates observed in periodicallydriven cavity were compared with standard lid-driven cavity and found to be more. From experiments as well as CFD simulations it can be observed that the amplitude shows strong effect on mixing than the frequency of plate motion.

\section{References}

[1] Chien, W. L., Rising, H., and Ottino, J. M., 1986 "Laminar and chaotic mixing in several cavity flows," Journal of Fluid Mechanics, 170, pp. 355377.

[2] C.W. Leong, J.M. Ottino, 1989 "Experiments on mixing due to chaotic advection in a cavity," Journal of Fluid Mechanics, 209, pp. 463-499.

[3] Anderson, P. D., Galaktionov, O. S., Peters, G. W. M., Van De Vosse, F. N., and Meijer, H. E. H., 1999, " Analysis of mixing in three-dimensional time-periodic cavity flows," Journal of Non-Newtonian Mechanics, 386, pp.149-169.

[4] Fountain, G. O., Khakhar, D. V., and Ottino. J. M., 1998, "Visualisation of three-dimensional chaos" Science, 281, pp. 683-686.

[5] Roberts, E. P.L., Mackley, M. R., 1995, "The simulation of stretch rates for the quantitative prediction and mapping of mixing within a channel flow," Chemical Engineering Science, 50, 3727-3746. 